\title{
Les jeunes Canadiens sont-ils toujours exposés à la fumée secondaire à la maison et en voiture?
}

\author{
A. Barisic, M.H.P. (1); S. T. Leatherdale, Ph. D. (2); R. Burkhalter, M. Math. (3); R. Ahmed, Ph. D. (4)
}

Cet article a fait l'objet d'une évaluation par les pairs.

\section{Résumé}

Introduction : Nous étudions dans cet article la prévalence de l'exposition à la fumée secondaire (FS) chez les jeunes à la maison et en voiture, les variations du niveau d'exposition à la FS dans le temps et les facteurs associés aux croyances des jeunes au sujet de l'exposition à la FS, grâce à un échantillon représentatif à l'échelle nationale des jeunes Canadiens.

Méthodologie : Nous avons mené une analyse descriptive de l'exposition à la FS à la maison et en voiture à l'aide des données de l'Enquête sur le tabagisme chez les jeunes (2004, 2006 et 2008). Une analyse de régression logistique a été effectuée afin d'analyser les facteurs associés aux croyances des jeunes au sujet de l'exposition à la FS en 2008.

Résultats : En 2008, 21,5 \% des jeunes ont signalé avoir été exposés à la FS à la maison chaque jour ou presque chaque jour, tandis que 27,3\% des jeunes ont déclaré avoir été exposés à la FS à bord d'une voiture au moins une fois au cours de la semaine précédente. Entre 2004 et 2008, la prévalence de l'exposition quotidienne à la FS au domicile et en voiture a diminué respectivement de 4,7\% et de 18,0\%.

Conclusion : Malgré la diminution de l'exposition à la FS au fil du temps, un nombre important de jeunes Canadiens continuent à être exposés à la FS chez eux et en voiture. Il faut redoubler d'efforts pour mettre en œuvre et évaluer des politiques visant à protéger les jeunes de la FS.

Mots-clés : jeunes, pollution par la fumée du tabac, prévention, voiture/véhicule, foyer/ménage

\section{Introduction}

La fumée secondaire (FS) désigne le mélange de contaminants libérés dans l'air par la combustion du tabac qui brûle et par la fumée exhalée par le fumeur ${ }^{1}$. Les effets nocifs associés à la FS ont été largement documentés, et comprennent un risque accru de maladies cardiovasculaires et de plusieurs cancers ${ }^{2}$. De nouvelles données probantes selon lesquelles les enfants sont particulièrement vulné- rables aux effets négatifs sur la santé associés à l'exposition à la FS suscitent d'autant plus des préoccupations ${ }^{2}$. Les enfants ont un système immunitaire moins développé, ils respirent plus rapidement et, en raison de leur petite taille, absorbent une plus grande quantité de polluants ${ }^{3}$. L'exposition à la FS chez les jeunes a ainsi été associée à un risque accru d'infections des voies respiratoires inférieures, comme la bronchite et la pneumonie, d'irritation des voies respira- toires supérieures, d'asthme, d'accumulation de liquide dans l'oreille moyenne, de syndrome de mort subite du nourrisson et de diminution de la fonction pulmonaire ${ }^{1,4-6}$. De plus, l'exposition à la FS chez les jeunes a été associée à une augmentation du nombre de journées avec activité réduite, alitement et absentéisme scolaire ${ }^{7}$. D’autres données laissent penser que les jeunes exposés à la FS risquent davantage de devenir fumeurs à leur tour ${ }^{8}$.

Malgré les données probantes démontrant les dangers de l'exposition à la FS dans les véhicules $^{9}$, Leatherdale et Ahmed $^{10}$ ont établi, à l'aide des données de l'Enquête sur le tabagisme chez les jeunes (ETJ) de 2004, que 26,3 \% des jeunes Canadiens avaient été exposés à la FS à bord d'un véhicule au moins une fois au cours de la semaine précédent l'enquête. De même, 23,1 \% des jeunes Canadiens avaient été exposés à la FS chez eux, et ce, malgré le fait que la grande majorité des jeunes aient déclaré que fumer devrait être interdit dans les véhicules et les maisons où il y a des enfants ${ }^{10}$. Cette disparité montre que les jeunes sont sans doute incapables d'éviter dans certains contextes d'être exposés à la FS ou de limiter cette exposition.

Cette étude a pour objet d'analyser la fréquence de l'exposition des jeunes à la FS chez eux et en voiture, les croyances des jeunes au sujet du tabagisme en présence d'enfants dans ces contextes, l'évolution de la prévalence de l'exposition à la FS dans ces contextes et enfin l'évolution entre 2004 et 2008 des croyances au sujet du tabagisme.

Rattachement des auteurs :

1. Prévention et lutte contre le cancer, Action Cancer Ontario, Toronto (Ontario), Canada

2. École de santé publique et de systèmes de soins de santé, Université de Waterloo, Waterloo (Ontario), Canada

3. Centre pour l'avancement de la santé des populations Propel, Université de Waterloo et Société canadienne du cancer, Waterloo (Ontario), Canada

4. Épidémiologie et registre sur le cancer, Action cancer Manitoba, Winnipeg (Manitoba), Canada

Correspondance : Scott T. Leatherdale, École de santé publique et de systèmes de soins de santé, Université de Waterloo, 200, Avenue University West, BMH 1038, Waterloo (Ontario) N2L 3G1; tél. : 519-888-4567 poste 37812; courriel : sleather@uwaterloo.ca 


\section{Méthodologie}

Cette étude s'est appuyée sur des données représentatives à l'échelle nationale recueillies dans le cadre des vagues de l'ETJ de 2004, 2006 et 2008. Des renseignements détaillés sur le plan d'échantillonnage, les méthodes employées et les taux pour chaque vague de l'ETJ sont disponibles en version imprimée ${ }^{11-13}$ et en ligne (www.yss.uwaterloo. ca). En résumé, les populations cibles pour les trois vagues étaient de jeunes résidents canadiens inscrits dans les niveaux scolaires appropriés et fréquentant une école publique ou privée dans l'une des 10 provinces du Canada. Les résidents du Yukon, du Nunavut et des Territoires du Nord-Ouest ont été exclus des populations cibles, tout comme les jeunes vivant en établissement ou dans une réserve des Premières Nations et ceux fréquentant une école spécialisée ou une école au sein d'une base militaire. Nous avons recueilli les données dans le cadre d'une enquête de 30 à 40 minutes menée en classe auprès d'un échantillon représentatif d'établisse- ments scolaires et d'élèves. La principale différence liée au plan d'échantillonnage des trois vagues de l'ETJ portait sur les niveaux scolaires visés : l'ETJ de 2004 s'adressait aux élèves de la $5^{\mathrm{e}}$ à la $9^{\mathrm{e}}$ année, l'ETJ de 2006 à ceux de la $5^{\mathrm{e}}$ à la $12^{\mathrm{e}}$ année et l'ETJ de 2008 à ceux de la $6^{\mathrm{e}}$ à la $12^{\mathrm{e}}$ année. Dans cet article, nous avons utilisé les données des répondants de la $6^{\mathrm{e}}$ à la $9^{\mathrm{e}}$ année pour analyser l'évolution de la prévalence de l'exposition à la FS au domicile et en voiture (2004, $\mathrm{n}=23$ 362; 2006, n = 33 955;2008, n = 31 249), et les données de l'échantillon complet de répondants à l'ETJ de 2008 (élèves de la $6^{\mathrm{e}}$ à la $12^{\mathrm{e}}$ année; $\mathrm{n}=51$ 922) pour la modélisation prédictive.

Chaque vague de l'ETJ a permis de recueillir des renseignements sur l'exposition à la FS au domicile et en voiture, les croyances au sujet du tabagisme dans ces contextes, les comportements liés au tabagisme, les caractéristiques individuelles des répondants et l'argent de poche. Les mesures utilisées sont uniformes pour l'ensemble des vagues de
l'ETJ et les autres études ${ }^{10,14}$. Les croyances au sujet du tabagisme ont été évaluées au moyen des questions suivantes : "Devrait-on être autorisé à fumer en présence d'enfants à la maison? » ( Oui » / « Non » / « Je ne sais pas »), et " Devrait-on être autorisé à fumer en présence d'enfants en voiture? » (« Oui » / " Non » / « Je ne sais pas »). Les répondants devaient également répondre aux questions suivantes : "Quelles sont les règles concernant le tabagisme chez toi? » (« Personne n'a le droit de fumer à l'intérieur de la maison » / " Seuls certains invités peuvent fumer dans la maison » / «On peut fumer uniquement dans certaines zones de la maison » / " On peut fumer partout chez moi ", " À part toi-même, combien de personnes fument à l'intérieur de ta maison chaque jour ou presque chaque jour? » (« Aucune »/ « 1 personne »/ « 2 personnes " / « 3 personnes ou plus ») et «Au cours des 7 derniers jours, combien de fois es-tu monté en voiture avec quelqu'un qui fumait la cigarette? » (" Aucun » / « 1 ou 2 jours » / « 3 ou 4 jours » / « 5 ou 6 jours » / « Les 7 jours »).

TABLEAU 1

Statistiques descriptives sur les jeunes Canadiens de la $6^{\mathrm{e}}$ à la $\mathbf{9}^{\mathrm{e}}$ année selon l'année où les données ont été recueillies, Canada

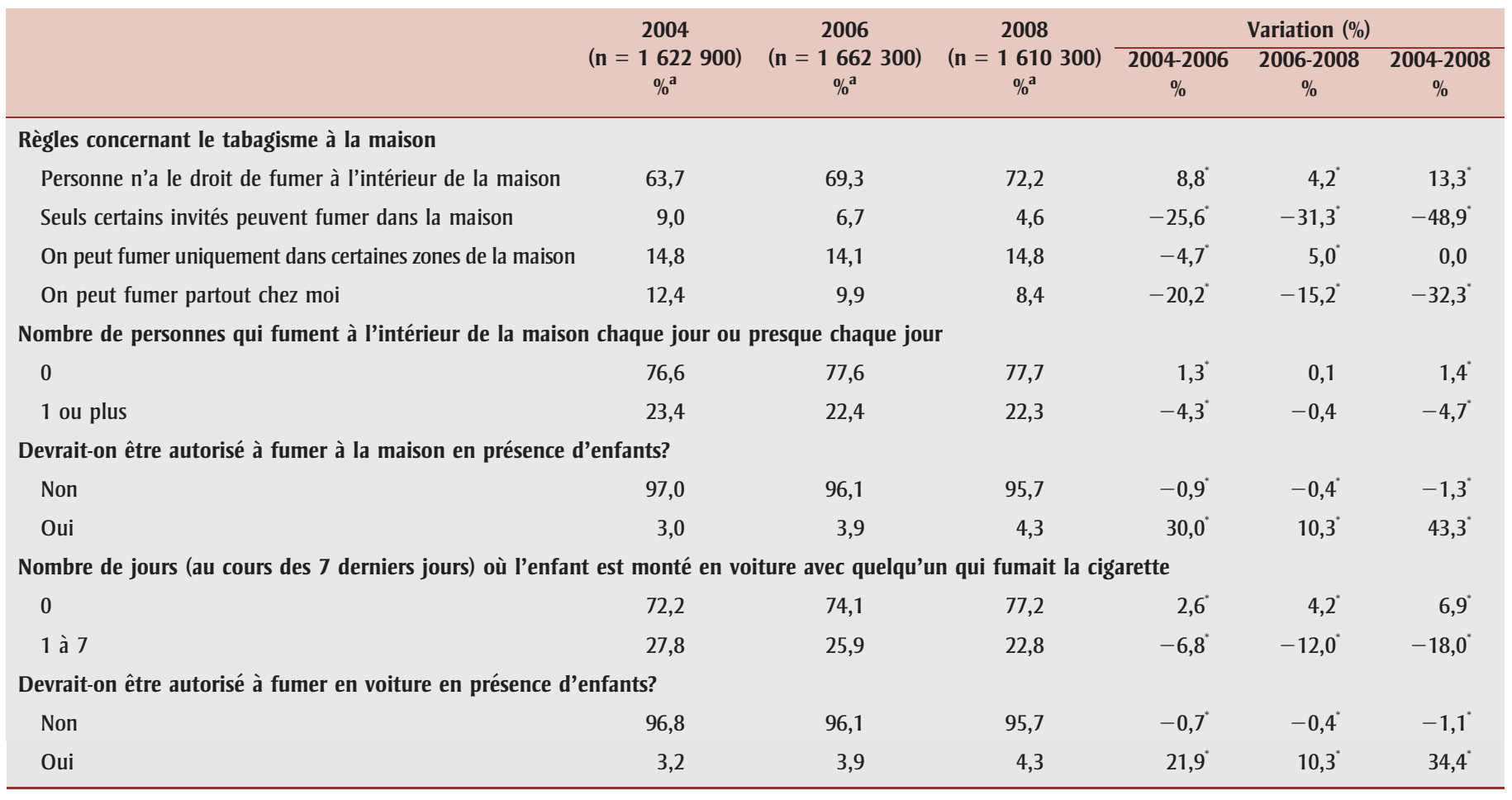

a Estimation pondérée de la population.

${ }^{*}$ Différence statistiquement significative, $p<0,05$. 


\section{Analyses statistiques}

À l'aide des données recueillies auprès des répondants de la $6^{\mathrm{e}}$ à la $12^{\mathrm{e}}$ année dans le cadre de l'ETJ de 2008, nous avons procédé à des analyses descriptives de l'exposition à la FS, des croyances au sujet du tabagisme, du statut tabagique et des caractéristiques individuelles selon le sexe. Nous avons utilisé pour cela des coefficients de pondération pour tenir compte de la non-réponse dans différentes provinces et différents groupes, ce qui a permis de réduire au minimum le biais différentiel dans les analyses en raison d'une variation du taux de réponse selon la région ou le groupe. Des modèles linéaires généralisés mixtes (à l'aide de PROC GLIMMIX pour SAS 9.2 [SAS Institute Inc., Cary, Caroline du Nord, États-Unis]) ont été utilisés avec les données non pondérées afin de vérifier si l'exposition à la FS au domicile ou en voiture était associée à des croyances de la part des jeunes au sujet du tabagisme en présence d'enfants, soit au domicile soit en voiture, après la prise en compte de variables sociodémographiques (sexe, statut de fumeur/non-fumeur, tabagisme parental et règles concernant le tabagisme à la maison) et après correction en fonction des regroupements au sein des écoles.

\section{Résultats}

Le tableau 1 présente les statistiques descriptives liées aux élèves de la $6^{\mathrm{e}}$ à la $9^{\mathrm{e}}$ année selon l'année où les données ont été recueillies (2004, 2006 et 2008). Les statistiques descriptives pour l'ensemble des élèves de la $6^{\mathrm{e}}$ à la $12^{\mathrm{e}}$ année pour 2008 sont présentées dans le tableau 2. La figure 1 montre l'évolution de la proportion de jeunes déclarant vivre dans une maison sans fumée, selon la région, tandis que la figure 2 indique l'évolution de la proportion de jeunes déclarant avoir été exposés au tabagisme à bord d'une voiture au cours des 7 derniers jours, selon la région. La figure 3 présente l'évolution de la prévalence de l'exposition au tabagisme à la maison et en voiture, selon le sexe, et la figure 4, la prévalence de l'exposition au tabagisme et les croyances des élèves de la $6^{\text {e }}$ à la $12^{\text {e }}$ année au sujet de l'exposition au tabagisme en 2008.
TABLEAU 2

Statistiques descriptives sur les jeunes Canadiens de la $6^{\mathrm{e}}$ année à la $12^{\mathrm{e}}$ année, selon le sexe, Canada, 2008

\begin{tabular}{|c|c|c|c|}
\hline & $\begin{array}{c}\text { Garçons } \\
(\mathrm{n}=1460300) \\
\%^{\mathrm{a}}\end{array}$ & $\begin{array}{c}\text { Filles } \\
(\mathrm{n}=1388 \text { 100) } \\
\%^{\mathrm{a}}\end{array}$ & $\begin{array}{c}\text { Total } \\
(\mathrm{n}=2848500) \\
\%^{\mathrm{a}}\end{array}$ \\
\hline \multicolumn{4}{|l|}{ Année scolaire } \\
\hline $6^{\mathrm{e}}$ & 13,1 & 13,6 & 13,3 \\
\hline $7^{e}$ & 13,8 & 14,2 & 14,0 \\
\hline $8^{\mathrm{e}}$ & 14,3 & 14,5 & 14,4 \\
\hline $9^{e}$ & 14,9 & 14,8 & 14,8 \\
\hline $10^{\mathrm{e}}$ & 15,5 & 14,8 & 15,2 \\
\hline $11^{\mathrm{e}}$ & 14,9 & 14,7 & 14,8 \\
\hline $12^{\mathrm{e}}$ & 13,6 & 13,4 & 13,5 \\
\hline \multicolumn{4}{|l|}{ Statut tabagique de l'enfant } \\
\hline N’a jamais fumé & 90,1 & 92,5 & 91,3 \\
\hline Fumeur & 8,9 & 6,4 & 7,7 \\
\hline Ex-fumeur & 0,9 & 1,1 & 1,0 \\
\hline \multicolumn{4}{|l|}{ Statut tabagique des parents } \\
\hline Aucun des parents ne fume & 56,1 & 57,0 & 56,5 \\
\hline Au moins l'un des parents fume & 43,9 & 43,0 & 43,5 \\
\hline \multicolumn{4}{|l|}{ Région } \\
\hline Canada atlantique ${ }^{\mathrm{b}}$ & 6,7 & 7,2 & 6,9 \\
\hline Québec & 19,3 & 19,4 & 19,4 \\
\hline Ontario & 41,4 & 40,5 & 40,9 \\
\hline Prairies $^{c}$ & 18,8 & 19,1 & 18,9 \\
\hline Colombie-Britannique & 13,8 & 13,9 & 13,9 \\
\hline \multicolumn{4}{|l|}{ Règles concernant le tabagisme à la maison } \\
\hline $\begin{array}{l}\text { Personne n'a le droit de fumer à l'intérieur de } \\
\text { la maison }\end{array}$ & 73,3 & 73,6 & 73,4 \\
\hline $\begin{array}{l}\text { Seuls certains invités peuvent fumer dans la } \\
\text { maison }\end{array}$ & 4,4 & 4,5 & 4,5 \\
\hline $\begin{array}{l}\text { On peut fumer uniquement dans certaines } \\
\text { zones de la maison }\end{array}$ & 13,7 & 14,1 & 13,9 \\
\hline On peut fumer partout chez moi & 8,6 & 7,8 & 8,2 \\
\hline
\end{tabular}

Nombre de personnes qui fument à l'intérieur de la maison chaque jour ou presque chaque jour

$\begin{array}{llll}0 & 78,3 & 78,7 & 78,5 \\ 1 \text { ou plus } & 21,7 & 21,3 & 21,5\end{array}$

Devrait-on être autorisé à fumer à la maison en présence d'enfants?

$\begin{array}{rrrr}\text { Non } & 93,2 & 97,2 & 95,2 \\ \text { Oui } & 6,8 & 2,8 & 4,8\end{array}$

Nombre de jours (au cours des 7 derniers jours) où l'enfant est monté en voiture avec quelqu'un qui fumait la cigarette

$\begin{array}{llll}0 & 73,0 & 72,4 & 72,7\end{array}$
1 à 7
27,0
27,6
27,3

Devrait-on être autorisé à fumer en voiture en présence d'enfants?

$\begin{array}{lrrr}\text { Non } & 93,2 & 97,2 & 95,2 \\ \text { Oui } & 6,8 & 2,8 & 4,8\end{array}$

a Estimation pondérée de la population.

b Nouveau-Brunswick, Île-du-Prince-Édouard, Nouvelle-Écosse, Terre-Neuve-et-Labrador.

c Alberta, Saskatchewan, Manitoba. 
FIGURE 1

Évolution de la proportion de jeunes de la $6^{\mathrm{e}}$ à la $9^{\mathrm{e}}$ année déclarant vivre dans un domicile sans fumée, selon la région, Canada, 2004, 2006 et 2008

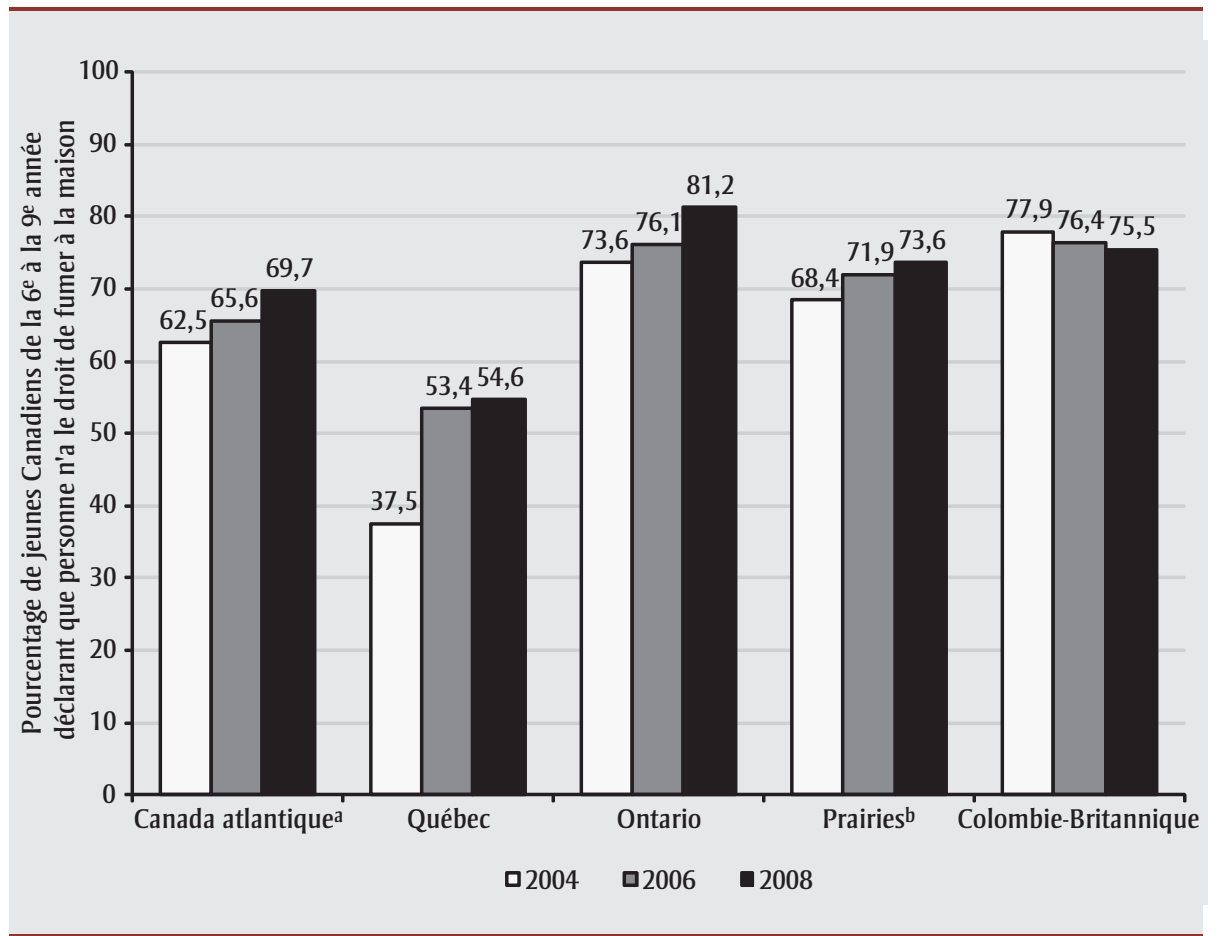

Source : Enquêtes sur le tabagisme chez les jeunes au Canada, 2004, 2006 et 2008

${ }^{a}$ Nouveau-Brunswick, île-du-Prince-Édouard, Nouvelle-Écosse, Terre-Neuve-et-Labrador.

${ }^{\mathrm{b}}$ Alberta, Saskatchewan, Manitoba.

\section{Exposition au tabagisme au domicile}

En 2008, environ le cinquième $(21,5 \%$; $\mathrm{n}=605300)$ des jeunes Canadiens de la $6^{\mathrm{e}}$ à la $12^{\mathrm{e}}$ année ont été exposés au tabagisme à la maison chaque jour ou presque chaque jour, avec des taux d'exposition similaires chez les garçons et les filles (tableau 2). En outre, 26,6 \% ( $\mathrm{n}=743200)$ des jeunes Canadiens ont déclaré que le tabagisme n'était pas complètement interdit à la maison, avec des taux similaires chez les garçons et les filles. Cependant, ces taux étaient différents selon les provinces $\left(\chi^{2}=2959,6 ; d l=12 ; p<0,0001\right):$ l'Ontario comptait le pourcentage le plus élevé de répondants ayant indiqué que le tabagisme était complètement interdit à la maison (80\%), tandis que le Québec faisait état du pourcentage le plus faible de domiciles sans fumée (56,1 \%; données non présentées).

Entre 2004 et 2008, la proportion d'élèves de la $6^{\mathrm{e}}$ à la $9^{\mathrm{e}}$ année indiquant que personne n'est autorisé à fumer à la maison avait augmenté de 13,3 \% (tableau 1). De plus, la prévalence des jeunes ayant déclaré que l'on peut fumer dans n'importe quelle pièce du domicile avait diminué de $32,3 \%$. Cependant, la proportion de jeunes ayant déclaré être exposés à la FS à la maison chaque jour ou presque chaque jour n'a diminué que de $4,7 \%$. De plus, cette diminution n’a été observée que chez les filles, la proportion augmentant chez les garçons. Dans toutes les provinces sauf en ColombieBritannique, il y a eu, au fil du temps, une augmentation de la proportion de jeunes ayant signalé ne pas être exposés à la FS à la maison (figure 1).

\section{Exposition au tabagisme en voiture}

En 2008, plus du quart $(27,3 \%$; $\mathrm{n}=716$ 500) des élèves de la $6^{\mathrm{e}}$ à la $12^{\mathrm{e}}$ année sont montés en voiture, au cours des 7 derniers jours, avec quelqu'un qui fumait la cigarette, les garçons et les filles ayant déclaré des taux similaires d'exposition. Cependant, les taux différaient d'une province à l'autre $\left(\chi^{2}=1138,1 ; d l=4\right.$; $p<0,0001)$, l'Ontario affichant la proportion la plus faible $(20,1 \%)$ et le Québec, la proportion la plus élevée (37,5 \% ; données non présentées).

La proportion d'élèves de la $6^{\mathrm{e}}$ à la $9^{\mathrm{e}}$ année ayant déclaré avoir été exposés à la FS en voiture a connu un déclin de $18 \%$ entre 2004 et 2008 (tableau 1) dans toutes les provinces, à l'exception du Québec et de la Colombie-Britannique (figure 2).

\section{Croyances au sujet du tabagisme à la maison}

En 2008, la majorité (95,2 \%; $\mathrm{n}=2473900$ ) des élèves de la $6^{\mathrm{e}}$ à la $12^{\mathrm{e}}$ année étaient d'avis que le tabagisme ne devrait pas être autorisé à la maison en présence d'enfants (tableau 2). Cette croyance était plus répandue chez les filles $(97,2 \%)$ que chez les garçons $(93,2 \%)$ $\left(\chi^{2}=420,4 ; d l=1 ; p<0,0001\right)$ et variait également selon la province $\left(\chi^{2}=127,8\right.$; $d l=4 ; p<0,0001)$, étant plus répandue chez les jeunes de l'Ontario (96,3\%) et moins répandue chez ceux du Québec (93,4 \%; données non présentées).

Chez les jeunes des deux sexes combinés, la proportion d'élèves de la $6^{\mathrm{e}}$ à la $9^{\mathrm{e}}$ année estimant que le tabagisme ne devrait pas être autorisé à la maison en présence d'enfants a accusé une légère baisse (1,3\%) entre 2004 et 2008 .

\section{Croyances au sujet du tabagisme en voiture}

En 2008, la majorité (95,2 \%; $\mathrm{n}=2484900)$ des élèves de la $6^{\mathrm{e}}$ à la $12^{\mathrm{e}}$ année étaient d'avis que le tabagisme ne devrait pas être autorisé en voiture en présence d'enfants (tableau 2). Cette croyance était plus répandue chez les filles $(97,2 \%)$ que chez les garçons $(93,2 \%)$ $\left(\chi^{2}=419,5 ; d l=1 ; p<0,0001\right)$ et variait également selon la province $\left(\chi^{2}=133,5\right.$; $d l=4 ; p<0,0001)$, étant plus fréquente chez les jeunes de l'Ontario (96,4\%) et moins fréquente chez ceux du Québec (93,5 \%; données non présentées).

Chez les jeunes des deux sexes combinés, la proportion d'élèves de la $6^{\mathrm{e}}$ à la $9^{\text {e }}$ année estimant que le tabagisme ne devrait pas être autorisé en voiture en 
FIGURE 2

Évolution de la proportion de jeunes de la $6^{\mathrm{e}}$ à la $9^{\mathrm{e}}$ année déclarant avoir été exposés au tabagisme à bord d'une voiture au cours des 7 derniers jours, selon la région, Canada, 2004, 2006 et 2008

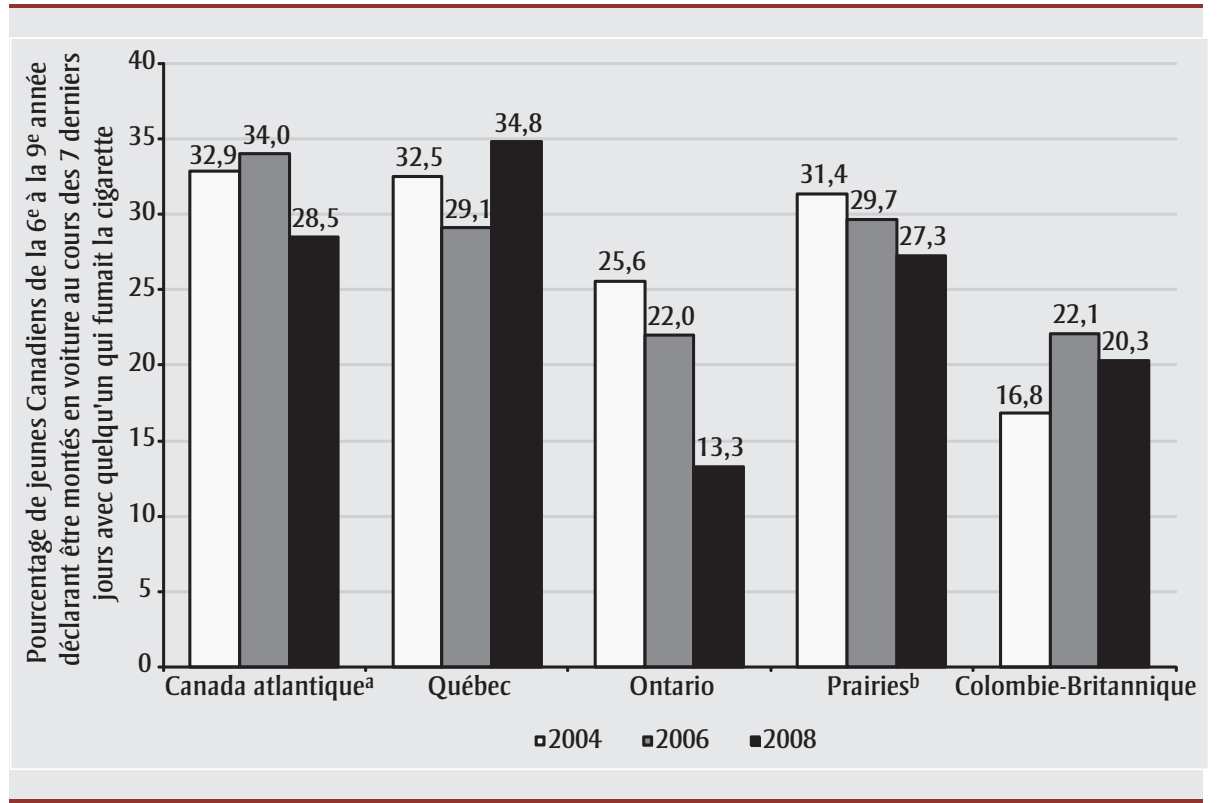

Source : Enquêtes sur le tabagisme chez les jeunes au Canada, 2004, 2006 et 2008 ${ }^{11,12,13}$.

${ }^{a}$ Nouveau-Brunswick, île-du-Prince-Édouard, Nouvelle-Écosse, Terre-Neuve-et-Labrador.

${ }^{\mathrm{b}}$ Alberta, Saskatchewan, Manitoba.

présence d'enfants a accusé un léger recul $(1,1 \%)$ entre 2004 et 2008 .

\section{Facteurs associés aux croyances au sujet $d u$ tabagisme à la maison}

En 2008 , les garçons de la $6^{\mathrm{e}}$ à la $12^{\mathrm{e}}$ année étaient plus nombreux que les filles à déclarer que le tabagisme ne devrait pas être autorisé à la maison en présence d'enfants (rapport de cotes $[\mathrm{RC}]=2,43$; intervalle de confiance [IC] à $95 \%: 2,20$ à
2,69; tableau 3). Tant les ex-fumeurs $(\mathrm{RC}=1,82$; IC à $95 \%: 1,23$ à 2,71) que les jeunes n'ayant jamais fumé $(\mathrm{RC}=4,26$; IC à $95 \%: 3,78$ à 4,79 ) étaient plus nombreux que les fumeurs à déclarer que le tabagisme ne devrait pas être autorisé à la maison en présence d'enfants. Comparativement aux jeunes qui vivaient dans un domicile où le tabagisme était complètement interdit, les jeunes qui avaient indiqué vivre dans un domicile où le tabagisme était permis étaient également plus nombreux à déclarer que le tabagisme ne devrait pas être autorisé à la maison en présence d'enfants ( $\mathrm{RC}=1,71$; IC à $95 \%$ : 1,50 à 1,96). De même, comparativement aux jeunes qui n'avaient signalé aucune exposition à la FS en voiture au cours de la semaine précédent l'enquête, ceux qui avaient indiqué être montés en voiture avec quelqu'un qui fumait la cigarette étaient plus nombreux à juger que le tabagisme ne devrait pas être autorisé à la maison en présence d'enfants ( $\mathrm{RC}=2,04$; IC à $95 \%: 1,81$ à 2,29).

\section{Facteurs associés aux croyances au sujet du tabagisme en voiture}

En 2008, les garçons de la $6^{\mathrm{e}}$ à la $12^{\mathrm{e}}$ année étaient plus nombreux que les filles à déclarer que le tabagisme ne devrait pas être autorisé en voiture en présence d'enfants $(\mathrm{RC}=2,58$; IC à $95 \%: 2,33$ à $2,85)$. Tant les ex-fumeurs ( $\mathrm{RC}=2,02$; IC à $95 \%: 1,39$ à 2,94$)$ que les jeunes n'ayant jamais fumé ( $\mathrm{RC}=4,14$; IC à $95 \%: 3,68$ à 4,65) étaient plus nombreux que les fumeurs à déclarer que le tabagisme ne devrait pas être autorisé en voiture en présence d'enfants. Les jeunes qui avaient indiqué vivre dans un domicile où le tabagisme était permis étaient également plus nombreux que les jeunes vivant dans un domicile où le tabagisme était complètement interdit à déclarer que le tabagisme ne devrait pas être autorisé en voiture en présence d'enfants ( $\mathrm{RC}=1,59$; IC à $95 \%: 1,39$ à 1,82). De même, comparativement aux jeunes qui n'avaient signalé aucune exposition à la FS en voiture au cours de la semaine précédent l'enquête,

FIGURE 3

Évolution de la prévalence de l'exposition au tabagisme à la maison et en voiture chez les jeunes de la $6^{\mathrm{e}}$ à la $9^{\mathrm{e}}$ année, selon le sexe, Canada, 2004, 2006 et 2008
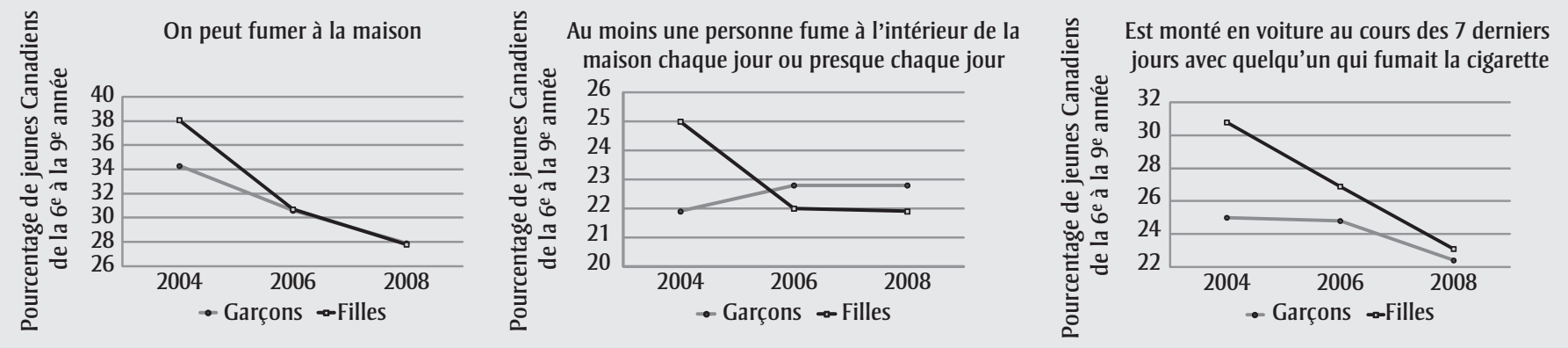

Source : Enquêtes sur le tabagisme chez les jeunes au Canada, 2004, 2006 et $2008^{11,12,13}$. 


\section{FIGURE 4}

Prévalence de l'exposition au tabagisme et croyances des jeunes de la $6^{\mathrm{e}}$ à la $12^{\mathrm{e}}$ année au sujet du tabagisme à la maison et en voiture, Canada, 2008

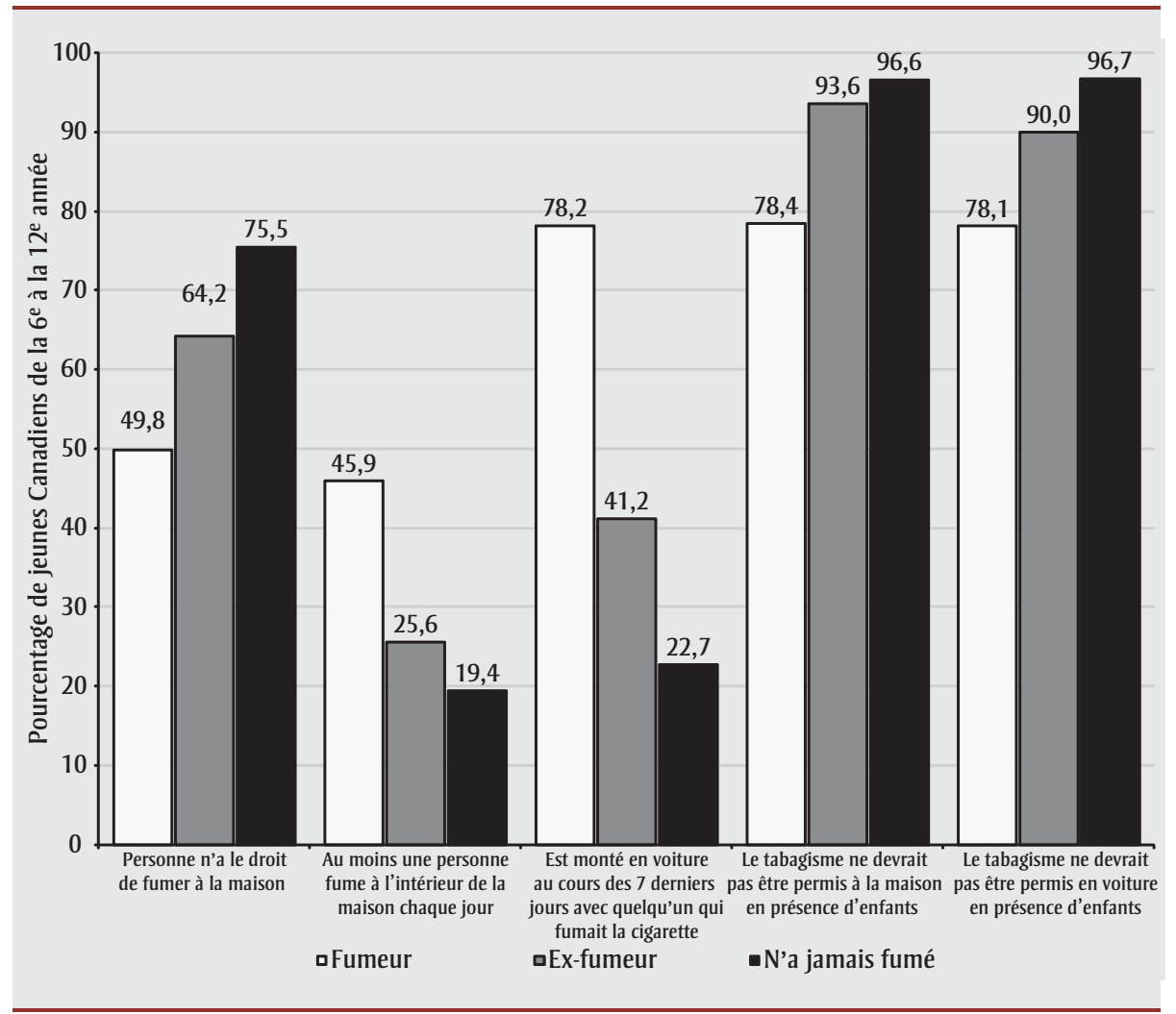

Source : Enquête sur le tabagisme chez les jeunes au Canada, $2008^{11}$.

les jeunes qui avaient indiqué être montés en voiture avec quelqu'un qui fumait la cigarette étaient plus nombreux à juger que le tabagisme ne devrait pas être autorisé en voiture en présence d'enfants $(\mathrm{RC}=2,73$; IC à $95 \%: 2,42$ à 3,07).

\section{Analyse}

Notre étude a révélé que, même si la proportion de jeunes exposés à la FS chez eux et en voiture a diminué entre 2004 et 2008, un nombre important de jeunes continuaient à être régulièrement exposés à la FS. La majorité des jeunes continuait à déclarer que le tabagisme ne devrait pas être permis en présence d'enfants dans ces contextes, les jeunes exposés à la FS chez eux ou en voiture étant plus nombreux à le faire.

Il s'avère que les jeunes pourraient être incapables d'éviter d'être exposés à la FS ou de limiter cette exposition dans certains contextes, ce qui laisse croire que, même s'il existe de nombreux programmes visant à protéger les enfants des dangers associés à l'exposition à la FS à la maison et en voiture, il est nécessaire de mettre en place des programmes ou des politiques plus solides et efficaces en ce domaine. Par exemple, en 2008, l'Association pulmonaire du Canada a lancé la campagne médiatique Fini, la fumée en auto! Nos enfants méritent bien ça!, une initiative visant à sensibiliser les parents à cette question et à aider la population canadienne à inciter les gouvernements provinciaux respectifs à promulguer une loi interdisant le tabagisme en voiture en présence d'enfants ${ }^{15}$. De nouvelles initiatives ont également été élaborées pour diminuer l'exposition à la FS dans les habitations à logements multiples (p. ex. appartements, condominiums, coopératives d'habitations, maisons en rangée). Un site Internet national tenu par SmokeFree Housing Canada fournit des données et fait la promotion de l'accès à des logements sans fumée à l'échelle du pays ${ }^{16}$. Jusqu'à présent, aucune province n’a promulgué de loi interdisant le taba- gisme dans les habitations à logements multiples, mais les propriétaires sont autorisés par la loi à adopter des politiques visant à interdire l'usage du tabac dans leurs habitations à logements multiples. S'il est vrai que le nombre de logements sans fumée est limité, des données non scientifiques incitent à penser que la demande pour ce type de logements est en hausse. Par exemple, en 2006, le plus grand propriétaire foncier du Manitoba, Globe General Agencies, a mis en œuvre une politique sans fumée s'appliquant à l'ensemble de ses 60 immeubles et incluant les logements eux-mêmes, les terrasses et les balcons ${ }^{17}$. Les répercussions de ces politiques sur l'exposition des enfants à la FS n'ont pas encore été étudiées.

À la lumière de nos résultats, qui indiquent que les jeunes pourraient être incapables d'éviter d'être exposés à la FS ou de limiter cette exposition dans certains contextes, et compte tenu du fait qu'il n'existe pas de niveau sécuritaire d'exposition à la $\mathrm{FS}^{2}$, il importe d'évaluer l'efficacité des programmes et des politiques visant à limiter l'exposition des jeunes à la FS. Il ressort des enquêtes " avant-après » de mesure de l'efficacité de la campagne La fumée secondaire à la maison et en voiture (2006-2007) que, sur l'ensemble des répondants se souvenant d'au moins l'une des publicités, $46 \%$ avaient déclaré avoir pris des mesures, ou prévoir en prendre à la suite de la campagne (ils avaient soit interdit le tabagisme à leur domicile ou dans leur voiture, soit convaincu les autres de ne pas fumer, soit eux-mêmes cessé de fumer $)^{18}$. Les enquêtes ont également révélé qu'un nombre moins élevé de répondants avait des idées fausses sur les façons de réduire l'exposition à la FS à la maison. Par exemple, la proportion de répondants qui estimaient que l'ouverture d'une fenêtre ou l'utilisation d'un ventilateur étaient des stratégies efficaces pour réduire l'exposition à la FS avait diminué de respectivement $17 \%$ et $10 \%{ }^{18}$. Cependant, même si les résultats de cette enquête sont prometteurs, il importe de poursuivre les travaux de recherche systématique pour mieux saisir les répercussions des mesures interdisant le tabagisme 
TABLEAU 3

Analyse de régression logistique examinant les facteurs associés aux croyances des jeunes Canadiens de la $6^{\mathrm{e}}$ à la $12^{\mathrm{e}}$ année au sujet de l'exposition à la FS à la maison et en voiture, Canada, 2008

\begin{tabular}{|c|c|c|}
\hline \multirow[t]{2}{*}{ Paramètres } & \multicolumn{2}{|c|}{$\begin{array}{l}\text { Rapport de cotes ajustéa } \\
\text { (IC à } 95 \%)\end{array}$} \\
\hline & $\begin{array}{c}\text { Modèle } 1 \\
\text { Le tabagisme ne devrait pas être permis à la } \\
\text { maison en présence d'enfants }\end{array}$ & $\begin{array}{l}\text { Modèle } 2 \\
\text { Le tabagisme ne devrait pas être permis en } \\
\text { voiture en présence d'enfants }\end{array}$ \\
\hline \multicolumn{3}{|l|}{ Sexe } \\
\hline Filles & 1,00 & 1,00 \\
\hline \multicolumn{3}{|l|}{ Statut tabagique de l'enfant } \\
\hline N’a jamais fumé & 1,00 & 1,00 \\
\hline Fumeur & $4,26(3,78 \text { à } 4,79)^{* *}$ & $4,14(3,68 \text { à } 4,65)^{* *}$ \\
\hline Ex-fumeur & $1,82(1,23 \text { à } 2,71)^{\circ}$ & $2,02(1,39 \text { à } 2,94)^{* *}$ \\
\hline \multicolumn{3}{|l|}{ Statut tabagique des parents } \\
\hline Personne n’a le droit de fumer à l'intérieur de la maison & 1,00 & 1,00 \\
\hline On a le droit de fumer dans la maison & $1,71(1,50 \text { à } 1,96)^{*}$ & $1,59(1,39 \text { à } 1,82)^{*}$ \\
\hline \multicolumn{3}{|c|}{ Nombre de personnes qui fument à l'intérieur de la maison chaque jour ou presque chaque jour } \\
\hline 0 & 1,00 & 1,00 \\
\hline 1 ou plus & $1,40(1,21 \text { à } 1,62)^{*}$ & $1,29(1,11 \text { à } 1,49)^{* *}$ \\
\hline \multicolumn{3}{|c|}{ Nombre de jours (au cours des 7 derniers jours) où l'enfant est monté en voiture avec quelqu'un qui fumait la cigarette } \\
\hline 0 & 1,00 & 1,00 \\
\hline 1 à 7 & $2,04(1,81 \text { à } 2,29)^{*}$ & $2,73(2,42 \text { à } 3,07)^{-*}$ \\
\hline
\end{tabular}

Abréviations : FS, fumée secondaire; IC, intervalle de confiance.

Remarques : Modèle $1: 1=$ Non $(n=41317), 0=$ Oui ou Je ne sais pas $(n=2043)$.

Modèle $2: 1$ = Non $(n=41435), 0$ = Oui ou Je ne sais pas $(n=2111)$.

${ }^{a}$ Les rapports de cotes indiqués ont été ajustés pour toutes les autres variables dans le tableau ainsi que pour la région et le niveau scolaire.

${ }^{*} p<0,01$.

${ }^{* *} p<0,001$.

à la maison en vue de réduire l'exposition des jeunes à la FS.

Il faudra également mener d'autres travaux de recherche pour déterminer l'efficacité des politiques visant à réduire l'exposition des jeunes à la FS en voiture. En effet, bien que toutes les provinces canadiennes, à l'exception du Québec, aient adopté des lois interdisant l'usage du tabac dans les véhicules en présence d'enfants ${ }^{19}$, nous avons recensé une seule étude canadienne portant sur les répercussions possibles de ces politiques. Dans son étude comparant l'exposition des jeunes à la FS avant et après l'adoption de ces lois à l'aide des données de l'Enquête de surveillance de l'usage du tabac au Canada (ESUTC) et de l'ETJ, Nguyen ${ }^{20}$ a constaté une diminution respective de $10 \%$ et de $26 \%$ de l'exposition des enfants à la FS à bord des voitures. Même si les résultats de cette étude sont encourageants, comme les données présentées sont fondées sur des études transversales, elles ne fournissent pas véritablement de données probantes solides quant à l'efficacité de ces politiques. Il serait utile pour la communauté de pratique et de recherche que des travaux de recherche pertinents soient menés selon un modèle de recherche longitudinale pour déterminer les répercussions des nouvelles politiques lorsqu'elles sont mises en pratique. Autrement dit, il faudrait utiliser des expériences réalisées dans des conditions naturelles pour produire des données probantes fondées sur la pratique ${ }^{21}$.
Dans l'étude susmentionnée, Nguyen ${ }^{20}$ n'a pas trouvé de données à l'appui d'un usage du tabac au domicile compensant l'interdiction de fumer dans les véhicules, mais des études antérieures avaient fait état d'une augmentation du tabagisme à la maison après l'entrée en vigueur de l'interdiction de fumer dans les lieux publics ${ }^{22}$. Compte tenu du fait que le domicile est l'un des rares endroits où l'usage du tabac est toujours permis, il importe d'effectuer une analyse approfondie des répercussions, sur l'exposition des enfants à la FS par effet de compensation, de la loi récemment adoptée dans la plupart des provinces interdisant le tabagisme dans les véhicules. De telles analyses auront des répercussions importantes sur la santé publique en ce qui concerne les politiques et les programmes 
visant à diminuer l'exposition des enfants à la FS, et pourraient également influer sur les futures politiques et initiatives ayant pour but de diminuer l'exposition à la FS au domicile.

Il ressort également de notre étude que, bien que la proportion globale de jeunes exposés à la FS chez eux et en voiture ait diminué au fil du temps, les filles ont été plus nombreuses à faire état d'une diminution de l'exposition que les garçons (figure 3). En fait, la proportion de garçons exposés à la FS chez eux chaque jour ou presque chaque jour a plutôt augmenté entre 2004 et 2008. On ne s'explique pas cette divergence entre les deux sexes, mais, compte tenu du fait que le taux de tabagisme est plus élevé chez les garçons, il est probable que le risque que les parents aient exposé leurs enfants à la FS ait été plus important si ces derniers étaient eux-mêmes fumeurs. Il se peut également que les non-fumeurs (que l'on retrouve plus fréquemment chez les filles) aient insisté davantage pour vivre dans un domicile sans fumée. Les divergences entre sexes sont préoccupantes et des travaux de recherche additionnels seraient nécessaires pour mieux comprendre les mécanismes qui les sous-tendent. Ils fourniraient des renseignements utiles pour l'élaboration de programmes et de politiques ciblés visant à protéger les garçons de la FS.

En conclusion, il ressort de notre étude que, malgré le fait que la majorité des élèves ait déclaré que le tabagisme ne devrait pas être permis à la maison et en voiture en présence d'enfants, un grand nombre de jeunes Canadiens continuaient à être régulièrement exposés à la FS. Ces constatations laissent entendre que les jeunes pourraient être incapables d'éviter d'être exposés à la FS ou de limiter leur exposition, ce qui renforce la nécessité d'entreprendre des travaux de recherche pour analyser les programmes et les politiques susceptibles de réduire l'exposition des jeunes à la FS.

\section{Références}

1. U.S. Department of Health and Human Services. How tobacco smoke causes disease: the biology and behavioral basis for smoking-attributable disease: a report of the Surgeon General. Atlanta (GA) : U.S. Department of Health and Human Services, Centers for Disease Control and Prevention, National Center for Chronic Disease Prevention and Health Promotion, Office on Smoking and Health; 2010.

2. U.S. Department of Health and Human Services. The health consequences of involuntary exposure to tobacco smoke: a report of the Surgeon General. Atlanta (GA) : U.S. Department of Health and Human Services, Centers for Disease Control and Prevention, Coordinating Center for Health Promotion, National Center for Chronic Disease Prevention and Health Promotion, Office on Smoking and Health; 2006.

3. Bearer CF. Environmental health hazards: how children are different from adults. Future Child. 2005;5(2):11-26.

4. California Environmental Protection Agency. Proposed identification of environmental tobacco smoke as a toxic air contaminant. Part B: health effects. Sacramento (CA) : California Environmental Protection Agency, Office of Environmental Health Hazard Assessment; 2005.

5. World Health Organization International Agency for Research on Cancer. IARC monographs on the evaluation of carcinogenic risks to humans: tobacco smoke and involuntary smoking. Volume 83. Geneva (CH) : World Health Organization; 2004.

6. World Health Organization. International consultation on environmental tobacco smoke (ETS) and child health. Geneva (CH) : World Health Organization; 1999.

7. Mannino DM, Siegel M, Husten C, Rose D, Etzel R. Environmental tobacco smoke exposure and health effects in children: results from the 1991 National Health Interview Survey. Tob Control. 1996;5(1):13-18.

8. Centers for Disease Control and Prevention. Exposure to secondhand smoke among students aged 13-15 years--worldwide, 2000-2007. MMWR. 2007;56(20):497-500.
9. Sendzik T, Fong GT, Travers MJ, Hyland A. An experimental investigation of Tobacco smoke pollution in cars. Nicotine and Tob Res. 2009;11(6):627-34.

10. Leatherdale ST, Smith P, Ahmed R. Youth exposure to smoking in homes and cars: how often does it happen and what do you think about it? Tob Control. 2008;17(2):8692.

11. Santé Canada. Enquête sur le tabagisme chez les jeunes de 2008-2009. Ottawa (Ont.) : Santé Canada; 2010.

12. Santé Canada. Enquête sur le tabagisme chez les jeunes de 2006-2007. Ottawa (Ont.) : Santé Canada; 2008.

13. Santé Canada. Enquête sur le tabagisme chez les jeunes de 2004-2005. Ottawa (Ont.) : Santé Canada; 2007.

14. Leatherdale S, Ahmed R. Second-hand smoke exposure in homes and in cars among Canadian youth: current prevalence, beliefs about exposure, and changes between 2004 and 2006. Cancer Causes Control. 2009;20(6):855-65.

15. L'Association pulmonaire. Nos enfants le méritent bien : une campagne nationale de l'Association pulmonaire pour revendiquer que l'on ne fume plus en auto [Internet]. Ottawa (Ont.) : L'Association pulmonaire; 2008 [consultation le 11 mars 2011]. Consultable en ligne à la page : http://www .poumon.ca/media-medias/news-nouvelles _f.php?id = 101

16. Association pour les droits des non-fumeurs (ADNF). Second-hand smoke in multi-unit dwellings [Internet]. Toronto (Ont.) : ADNF; [consultation le 3 mars 2011]. Consultable en ligne à la page : http://www.nsra-adnf .ca/cms/page1433.cfm

17. Smoke-Free Housing Canada. What landlords, condominium owners and co-ops need to know [Internet]. Smoke-Free Housing Canada; 2007 [consultation le 3 mars 2011]. Consultable en ligne à la page: http://www.smokefreehousing.ca/what _owners_need_to_know.html

18. Kosir M, Gutierrez K. Lessons learned globally: secondhand smoke mass media campaigns. Saint Paul (MN) : Global Dialogue for Effective Stop Smoking Campaigns; 2009. 
19. Association pour les droits des nonfumeurs. Second-hand smoke in cars [Internet]. Toronto (Ont.) : ADNF; [consultation le 3 mars 2011]. Consultable en ligne à la page : http://www.nsra-adnf.ca /cms/page1497.cfm

20. Nguyen HV. Do smoke-free car laws work? Evidence from a quasi-experiment. J Health Econ. 2013;32(1):138-48.

21. Leatherdale ST. Evaluating school-based tobacco control programs and policies: an opportunity gained and many opportunities lost. Can J Prog Eval. 2012;24:91-108.

22. Adda J, Cornaglia F. The effects of bans and taxes on passive smoking. Am Econ J: App Econ. 2010;2(1):1-32. 